Review

\title{
NK Cell Metabolism and the Potential Offered for Cancer Immunotherapy
}

\author{
Clair M. Gardiner
}

School of Biochemistry and Immunology, Trinity Biomedical Sciences Institute, 152-160 Pearse Street, Trinity College, Dublin 2, Ireland;

Email: clair.gardiner@tcd.ie

\begin{abstract}
Immunotherapy may provide a future where curing metastatic cancer is not a goal but a reality. Significant progress has already been made with the clinical success of check-point inhibitors and more recently, CAR-T cells. It is likely that combination immune-therapies will be required for optimal success against cancer. It is also likely that conventional cell based approaches that focus on $\mathrm{T}$ cells may not provide expected dividends, and that harnessing the innate immune response might bring greater rewards. In this regard, Natural Killer (NK) cells, occasionally considered the innate counterpart of $\mathrm{T}$ cells, offer huge potential. This review considers how and why this may be so and discusses the current state of play with respect to NK cell therapies for cancer. Importantly, it will detail our current knowledge of NK cell metabolism and demonstrate how this information can synergise with the rapidly evolving field of immunometabolism, to provide new, exciting and effective ways to treat a range of cancer types.
\end{abstract}

KEYWORDS: NK cell; cancer; immunotherapy; metabolism; CAR-NK; adoptive cell therapy

\section{INTRODUCTION}

The use of immunotherapy to treat a wide range of diseases has had global impact and in 2018, eight of the top ten selling drugs globally were antibodies (creative-biolabs.com). Indeed, immunotherapy has heralded a new era for cancer treatments as evidenced by the recent Nobel prize awarded to two Immunologists for their work on basic $\mathrm{T}$ cell regulation, which was subsequently translated into the first checkpoint inhibitors in clinical use [1]. Another significant breakthrough for cancer immunotherapy is the advent of CAR-T adoptive cell therapies [2].

Most research efforts in this area to date have focused on $\mathrm{T}$ cells, reflecting the relative popularity of $\mathrm{T}$ cells in mainstream Immunology. These therapies have not been without their problems and cytokine release syndrome (CRS) has been commonly observed in T cell therapies, with occasional fatal results [3]. CRS is largely due to intrinsic features associated with $\mathrm{T}$ cell biology and significant patient management is

Received: 21 March 2019

Accepted: 06 June 2019

Published: 12 June 2019

Copyright (c) 2019 by the author(s). Licensee Hapres, London, United Kingdom. This is an open access article distributed under the terms and conditions of Creative Commons Attribution 4.0 International License. 
required. As a result, the tide is turning and there is increasing recognition that there are probably better possibilities and increased potential for innate immune cells, in particular Natural Killer (NK) cells, to have impact in this arena [4].

The potential of NK cells for immunotherapy has long been recognized. Over 40 years of accumulated evidence supports their key role in killing cancer cells in vivo where they are thought to be particularly important in the control of metastatic spread [5,6]. This has been extensively reviewed elsewhere (including [7]). Earliest efforts to harness NK cells focused on their activation in vivo using cytokines such as IL2 and although some anti-cancer efficacy was observed [8], toxicity associated with high dose of cytokine administration e.g., vascular leaking syndrome, precluded further development [9]. Today, however, NK cells are used in a variety of different ways and the rate of change from preclinical to clinical studies means that there is more immediate potential to translate promising findings into clinical trial. The potential to manipulate NK cells and improve their therapeutic activity depends on their specific clinical application and whether their use is primarily in an autologous (NK cells from patient themselves, can be manipulated either in vivo or ex vivo) or an allogeneic setting (NK cells from a third party e.g., donor, cell line).

In brief, most current autologous NK cell therapies use the natural ability of NK cells to recognize and kill antibody coated target cells by antibody dependent cellular cytotoxicity (ADCC). In clinical practice, targeted antibody therapies for solid tumours include anti-Her2 antibodies (e.g., Herceptin) for the treatment of Her2+ breast cancer, antiCD20 treatments (e.g., Rituximab) for lymphoma and anti-GD2 treatment for neuroblastoma [10]. While these therapies have had a significant impact, it is well known that NK cell effector functions deteriorate as cancer progresses [11] and thus, NK cell contributions to such immunotherapies are less optimal than they could be [12]. It was recently demonstrated that impaired metabolism was linked to the progressive NK dysfunction (though abnormal expression of fructose-1,6bisphosphatase enzyme)[11]. Understanding this immune dysfunction will help identify ways to improve current therapies.

NK cells are also used as a form of adoptive cell therapy and it is here that there is perhaps most potential for future therapies. A key benefit of adoptive cell therapy is that it allows manipulation of cells ex vivo using a variety of approaches and which can empower NK cells to survive and persist in vivo with enhanced effector functions. Adoptive NK cell therapies can use either autologous patient cells or a range of allogeneic NK (allo-NK) options [13]. The allo-NK cell options include healthy donor NK cells (with various matching options for KIR/HLA [14]), CAR-NK cells (e.g., cord blood [15] or iPSC derived [16]), NK cell lines (e.g., NK92), or indeed a combination of these (e.g., NK92-CAR-NK cells)[17]. Each of these NK cell sources are very different and manipulations for enhanced 
metabolism and function ex vivo will need to be tailored appropriately. For example autologous "exhausted" NK cells will have different metabolic or signaling requirements compared to iPSC derived CAR-NK cells.

However, understanding how the common mechanisms in which the immunosuppressive environment of cancer specifically impacts on NK cells will help to design strategies to equip both autologous and allogeneic adoptively transferred NK cells to survive and persist in a patient for maximum beneficial effect.

Most strategies to modulate human NK cells ex vivo have focused on cytokine stimulations although specific NK cell check-point inhibitors are also under development [4]. However, it is the area of immunometabolism, the focus of this review, that is likely to have most significant impact over the next few years.

\section{WHAT DO WE KNOW ABOUT NK CELL METABOLISM?}

Glucose is a key fuel used by NK cells [18] and is taken up through cellular receptors including Glut1. Once inside the cell, there are two key pathways by which glucose can be metabolized (see Figure 1). Glycolysis is a series of enzymatic steps which breaks down glucose into two molecules of pyruvate. Pyruvate can either be metabolized to lactate and secreted from the cell, or it can enter the mitochondria to be metabolized further by oxidative phosphorylation (Oxphos). Oxphos is the process by which electrons are transferred along the electron transport chain (ETC) machinery, and which culminates to drive ATP synthase and generate ATP. In general, generation of ATP through Oxphos is more efficient compared to glycolysis with respect to molecules of ATP generated per molecule of glucose. Both glycolysis and Oxphos are generally measured using extracellular metabolic flux analysers. These essentially measure $\mathrm{pH}$ changes and oxygen consumption which, in combination with specific metabolic inhibitors, are used to measure glycolytic flux and Oxphos of purified cell populations.

Quiescent, mature NK cells predominantly metabolise glucose by Oxphos [19]. However, when NK cells are activated by cytokine, they upregulate both glycolysis and Oxphos pathways [19-22]. This makes sense from a cellular perspective as during homeostasis, the primary requirement is to maintain existing cellular processes; thus Oxphos is very efficient in this regard. However, when the immune system is activated, it needs to increase both the number of immune cells present and to arm them with effector molecules required to carry out their specific effector functions. Thus, while increasing energy production by upregulating Oxphos is an obvious thing to do, upregulating glycolysis flux is also beneficial as, in addition to generating ATP, it also generates many biosynthetic precursors that are required to provide the biochemical building blocks for protein synthesis and proliferation. 


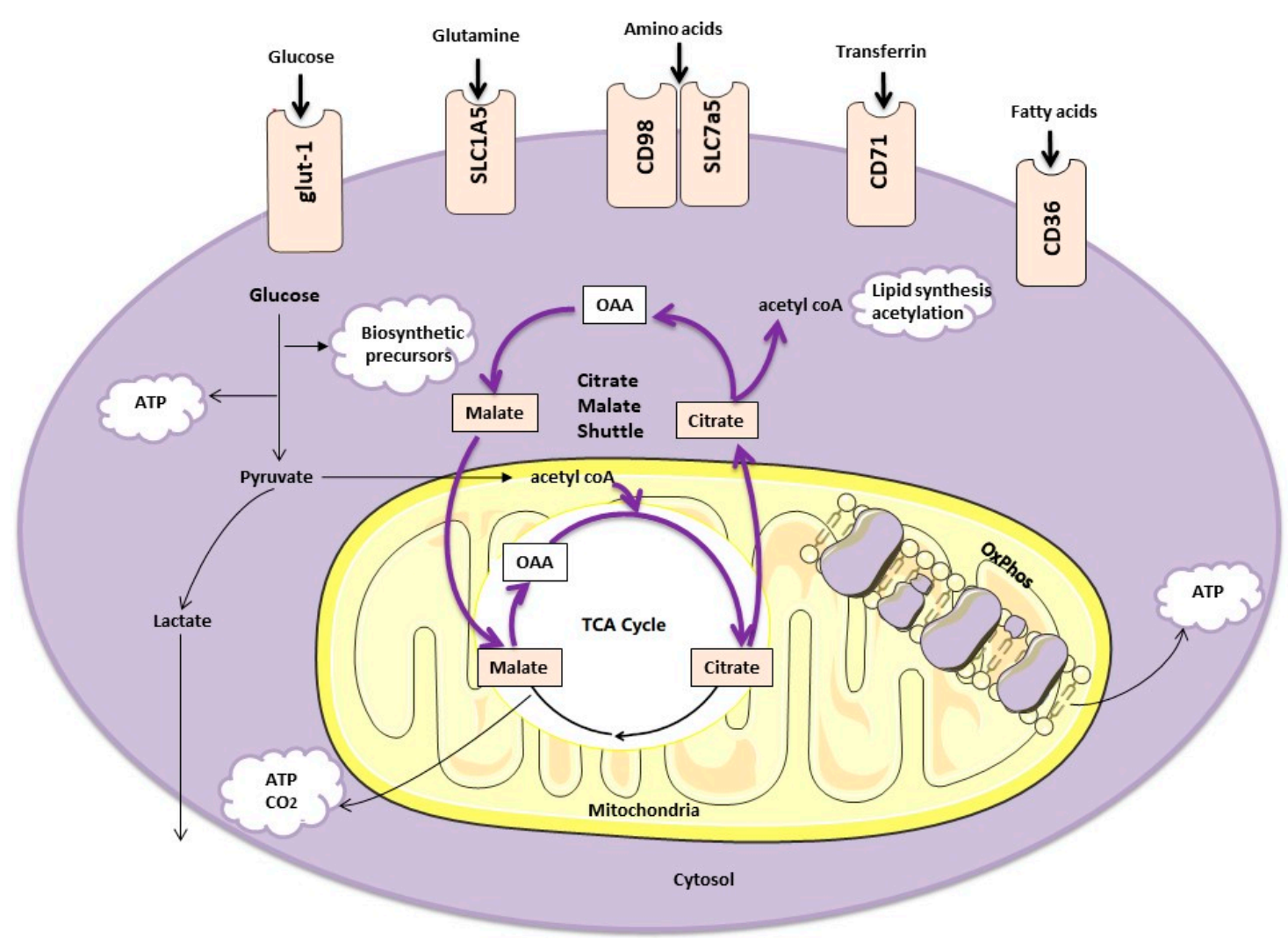

Figure 1. NK cell metabolism. NK cells primarily use glucose as a fuel. Activated NK cells increase expression of glut1 receptor which takes increased glucose into the cell. Activated NK cells also increase expression of other nutrient receptors including CD71 (transferrin receptor), SLC7a5 (import of large neutral amino acids), SLC1A5 (glutamine uptake) and CD36 (for free fatty acid uptake). Glucose is metabolized by glycolysis to yield pyruvate which is either further metabolized to lactate (and secreted) in the cytotosol, or converted to acetyl-Co-A for uptake into the mitochondria. It is initially converted to citrate as part of the normal TCA cycle. However, most of the citrate is then exported out of the mitochondria. It is processed by the ATP citrate lyase enzyme to generate acetyl-CoA (and oxaloacetate, OAA). Acetyl-Co-A in the cytosol is used for both lipid synthesis and acetylation reactions. The OAA generated is metabolized to malate which reenters the mitochondria, completing the cycle. This shuttle is known as the Citrate Malate Shuttle (CMS). The CMS can provide reducing equivalents that are required by the electron transport chain for the process of Oxidative Phosphoyrlation (Oxphos).

In line with this, uptake of nutrients from the environment is important for immune cells that are transitioning into effector cells [23]. This is also an obvious place where pathological changes, e.g., glucose deprivation in a tumour, or increased lipid availability during obesity, has the potential to impact on NK cell function. A number of nutrient receptors are routinely examined during immunometabolism analysis; these not only serve as an important correlate of increased metabolic activity but also provide important information about key nutrients utilised by NK cells. CD71 is the transferrin receptor. CD98 is an important co-receptor which works with some SLC transport family members for the uptake of large or neutral amino acids while glut 1 is the primary receptor used by activated lymphocytes to take glucose into 
cells. This field is in its infancy and these receptors represent the simplest first round of analysis that has been easily achieved by routine flow cytometry methods. As our knowledge expands, so too will investigations into the large range of molecules and receptors that are inevitably involved in regulating complex immune changes e.g., SLC7a5 (also known as LAT) [24], ferroportin (iron export receptor)[25] or CD36 (free fatty acid uptake).

\section{Specialised NK Cell Subsets Differ in Their Metabolic Profiles}

Many NK cell subsets have been defined based on phenotypic and functional differences [26]. For human NK cells, CD56 bright cells have classically been defined as being more responsive to cytokine with relatively higher levels of IFN $\gamma$ produced in response to cytokine, compared to $\mathrm{CD} 56^{\mathrm{dim}}$ cells. Within the $\mathrm{CD} 56^{\mathrm{dim}}$ subset, we can now also define canonical and adaptive NK cells (based on prior CMV exposure), licensed and unlicensed NK cells (based on cognate HLA/KIR receptor expression) and conventional NK cells (cNK) versus tissue resident NK cells (trNK). Evidence is mounting that each of these are characterized by metabolic configurations that may be important in regulating their functions, and indeed, their therapeutic potential.

A number of studies have demonstrated that CD56 ${ }^{\text {bright }}$ cells are more metabolically responsive, whether they are from peripheral blood or tissue resident [22,27]. While CD98 is expressed on all NK cells (and is upregulated in response to cytokine), CD71 is generally only present at low levels and increases preferentially on CD56 bright cells upon activation. The glutamine receptor (SLC1A5) is also preferentially upregulated on peripheral blood CD56 ${ }^{\text {bright }} \mathrm{NK}$ cells in response to cytokine [28].

There are also two studies within the last year that have defined licensed human NK cells as preferentially engaging glycolysis compared to unlicensed cells, even in a basal state. Using proteomic approaches, Schafer et al. reported that both glycolysis and Oxphos are used by licensed NK cells while unlicensed NK cell appears to depend exclusively on Oxphos, at least in terms of facilitating cytotoxicity [29]. It should be noted that most experiments were done on expanded NK cells and differences were not statistically significant when using primary cells. However, the suggestion that increased glycolysis is important for licensed cells is also supported by Pfeiffer et al., for KIR+ licensed NK cells [30]. That metabolic differences were also observed between KIR+ and NKG2A+ educated NK cells in this latter study suggests that metabolic differences resulting from alternative signaling pathways to evoke licensing may be possible.

Given the profound impact of CMV on NK cells, including the induction of phenotypic, signaling and functional differences, it is not surprising that the effects also extend to alterations in metabolism. While NK cells from HCMV seropositive donors had higher Oxphos (but not glycolysis) compared with seronegative donors, increases in both Oxphos 
and glycolysis were found in adaptive versus canonical cells from within a HCMV+ donor [31].

While murine subsets NK cell subsets are also known, data is only starting to emerge about potential metabolic differences. Of note, Filipovic et al. have recently published a comprehensive analysis of NK cell/ILC1 subsets in mouse uterus and liver [25]. In addition to phenotypic and functional differences, metabolic differences were also identified between trNK and cNK cells within the uterus. In addition to increased expression of receptors important for lactate and iron export, trNK cells also had higher expression of genes relating to cholesterol storage and homeostasis, compared to cNK. The authors speculated that CXCR6+ ILC1 cells in the uterus provided "innate" memory as they preferentially expanded in second pregnancies. As previous work on memory CD8 cells has defined a "futile fatty acid synthesis" pathway [32], this may be a good place to start. Irrespectively, these data provide strong evidence for specific metabolic signatures that can now be investigated at a cellular and biochemical level.

\section{NK Cells Adopt a Novel Metabolic Configuration}

While glucose is the key fuel for glycolysis, we know that Oxphos can be fuelled in a number of different ways. These include glucose, glutamine and fatty acids which can all feed into the TCA cycle and generate the reducing equivalents (NADH, FADH2) required for the Electron Transport Chain (ETC) and Oxphos. Any biochemistry text book will show the TCA cycle as the fundamental cellular pathway that fuels Oxphos and there was no reason to assume that this would be different in NK cells. One caveat of extracellular flux analysis is that it measures Oxphos by measuring oxygen consumption, and this is merely an endpoint in the pathway that does not reveal any of the biochemical events engaged to get to this point. It was therefore somewhat surprising when metabolic analysis of ${ }^{13} \mathrm{C}$-labelled glucose showed that the TCA cycle was not being used conventionally in NK cells [18]. Rather, the TCA intermediate, citrate, was predominantly exported out of the mitochondria. Furthermore, there was a lack of labeled carbon molecules in downstream TCA intermediates until malate, after which the labeled carbons were once again apparent. Citrate export from the mitochondria is important for the generation of acetyl-CoA that is required both for lipid synthesis and acetylation reactions within a cell. The resulting oxaloacetate (OAA) generated in the cytoplasm is converted to malate which is subsequently imported back into the mitochondria using the designated citrate malate obligate anti-porter. It appears that metabolically active NK cells have a high flux through this particular citrate malate shuttle. This shuttle can still supply reducing intermediates for the ETC (NADH), and Oxphos can continue but it has the additional output of acetyl-CoA. 
We can only speculate as to why NK cells adopt this configuration. It is possible that in addition to fueling Oxphos, acetyl-CoA is a particular metabolic requirement of NK cells. Indeed, there is a clear precedent in the literature that immune cells adopt specific metabolic configurations to facilitate their specialized functions. The two key roles of acetyl Co-A within a cell include its use as a substrate for lipid synthesis and for acetylation reactions. The role of acetyl Co-A is unlikely to be as a result of its use in lipid synthesis as inhibitors of the lipid synthesis pathway had no apparent effect on Oxphos [18]. However, acetyl-CoA is also used for acetylation reactions. Acetylation of proteins, generally on lysine residues, is an important post-translational modification that can regulate proteins in a myriad of ways e.g., affect nuclear translocation of transcription factors. Acetylation may be particularly important in metabolism as early studies showed that up to $35 \%$ of proteins within the mitochondria are acetylated [33]. However, it is acetylation of histones that has been best characterized as an important mechanism regulating chromatin accessibility and transcription of genes. The dynamic nature of histone acetylation/deacetylation is regulated by complementary enzyme families. In general, acetylation of lysines on the N-terminal tail of histones is associated with a more open chromatin structure and increased transcription [34]. The potential of these for regulating NK cell biology is under investigation.

\section{MECHANISMS REGULATING NK CELL METABOLISM}

Cytokines that activate NK cells clearly also drive metabolic reprogramming with increases in both glycolysis and Oxphos in NK cells reported. Some of the key regulators driving metabolic change include the transcriptions factors mTORC1, c-Myc and Srebp, and the transcriptional regulator ARID5B (reviewed elsewhere [35]). However, this field is only in its infancy and much basic biology awaits discovery. It is likely that NK cell metabolism is regulated at many levels including transcriptional, translational, post-translation and epigenetic levels. It will be through a detailed understanding of the mechanisms underpinning NK cell metabolism and function that we will best be able to arm NK cells for immunotherapy. Indeed, although IL15 is a strong driver of NK cell metabolic reprogramming, sustained and prolonged signaling during expansion of human cells for adoptive immunotherapy can actually result in an exhausted NK cell phenotype [36]. Care must be also taken in extrapolating experimental data from $\mathrm{T}$ cells as different receptors, including possible check-point inhibitors, cytokine responsiveness and metabolism distinguish these cell types.

Ourselves and others have reported that TGF $\beta$ can inhibit NK cell metabolism [37,38]. However, in the last year, two new mechanisms of negative regulation have been identified (see Figure 2). mTORC1 is the most consistently defined positive regulator of NK cell metabolism to date. mTOR is a highly conserved serine/threonine kinase. It, along with 
mLST8, forms either mTORC1 or mTORC2, depending on whether the complex further associates with either Raptor or Rictor subunit respectively. Several reports indicate that mTORC1 and mTORC2 are important during NK cell development [39-41] but a new role for mTORC2 in negative regulation of metabolism was also discovered [41]. Specific deletion of mTORC2 at the terminal stage of NK cell differentiation (by deletion of Rictor using NKp46Cre mice) resulted in increased expression of nutrient receptors, CD71 and CD98 on NK cells, suggesting that they might be more metabolically active. This was further supported by observed changes in the mitochondria including increased mitochondrial mass, mtROS and mitochondrial membrane potential in cells lacking mTORC2. This study also identified cross talk between mTORC1 and mTORC2. IL15 induced mTORC1 activity supported mTORC2 activity; however, mTORC2 suppressed mTORC1 activity by reducing pSTAT5 and SLC7A5 expression [41]. Thus, while mTORC1 activity promotes NK cell metabolism, mTORC2 activity appears to inhibit it.

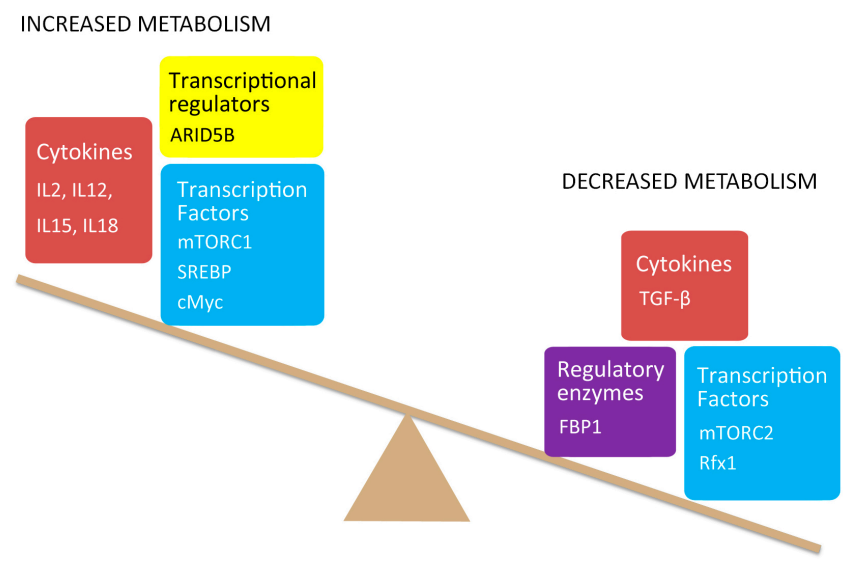

Figure 2. Known regulators of NK cell metabolism. Although a relatively new field, discoveries have been made regarding regulatory mechanism of NK cell metabolism. Amongst transcriptions factors that positively drive NK cell metabolism are mTORC1, c-Myc and Srebp while mTORC2 and Rfx1 have recently been described to negatively regulate the process. Cytokines that activate NK cells including IL2, IL15, IL12 and IL18 all positively drive metabolic reprogramming while TGF $\beta$ inhibits it. A small number of other regulators have been discovered thus far. These include ARID5B, a transcriptional regulator and FBP1, which negatively regulates glycolysis.

An unanticipated role for the transcription factor Rfx7 was discovered when expression profiles and knock-out phenotype revealed high levels in NK cells and a heightened metabolic response when Rfx7 was absent [42]. The data suggested that Rfx7 is a negative regulator of important metabolic activities including glycolysis, Oxphos and nutrient receptor expression (CD98). mTORC1 activity was increased in the absence of Rfx7 and limiting mTORC1 activity (using genetic tools) suggested that Rfx7 may function by negatively regulating mTORC1 signalling. However, as strong mTORC1 signalling, induced by IL15, could at least partially 
compensate for the Rfx7-/- phenotype, this suggested that Rfx7 might be more important when NK cells are not actively engaged in an immune response, i.e., Rfx7 might be more important in maintaining NK cell homeostasis.

Thus a common theme emerges where nutrient receptor expression is a key mechanism regulating NK cell metabolism, whether during homeostasis or activation [24,41,42]. Expression of receptors, in particular, SLC7a5, is increased through CD122 (IL15 or IL2) mediated signaling, and c-Myc is important in this process [24]. This axis can then be negatively regulated by Rfx7 (during homeostasis) and/or mTORC2 (during activation) and/or by modulation of both mTORC1 and p-STAT-5 signalling pathways [41,42].

\section{POTENTIAL MECHANISMS TO "INTRINSICALLY” IMPROVE NK CELLS FOR ADOPTIVE IMMUNOTHERAPY}

Adoptive cell therapy has a key advantage over in vivo manipulation of cells in terms of being able to genetically, pharmacologically or biologically manipulate NK cells to improve their effectiveness in vivo. The potential to engineer a perfect anti-cancer effector cell is there but a suite of solutions will probably be required, tailoring NK cells depending on the source of NK cell, cancer type, staging, time of intervention etc. A non-exhaustive list of potential interventions is shown in Figure 3 and where these relate to metabolism, they will be discussed briefly below.

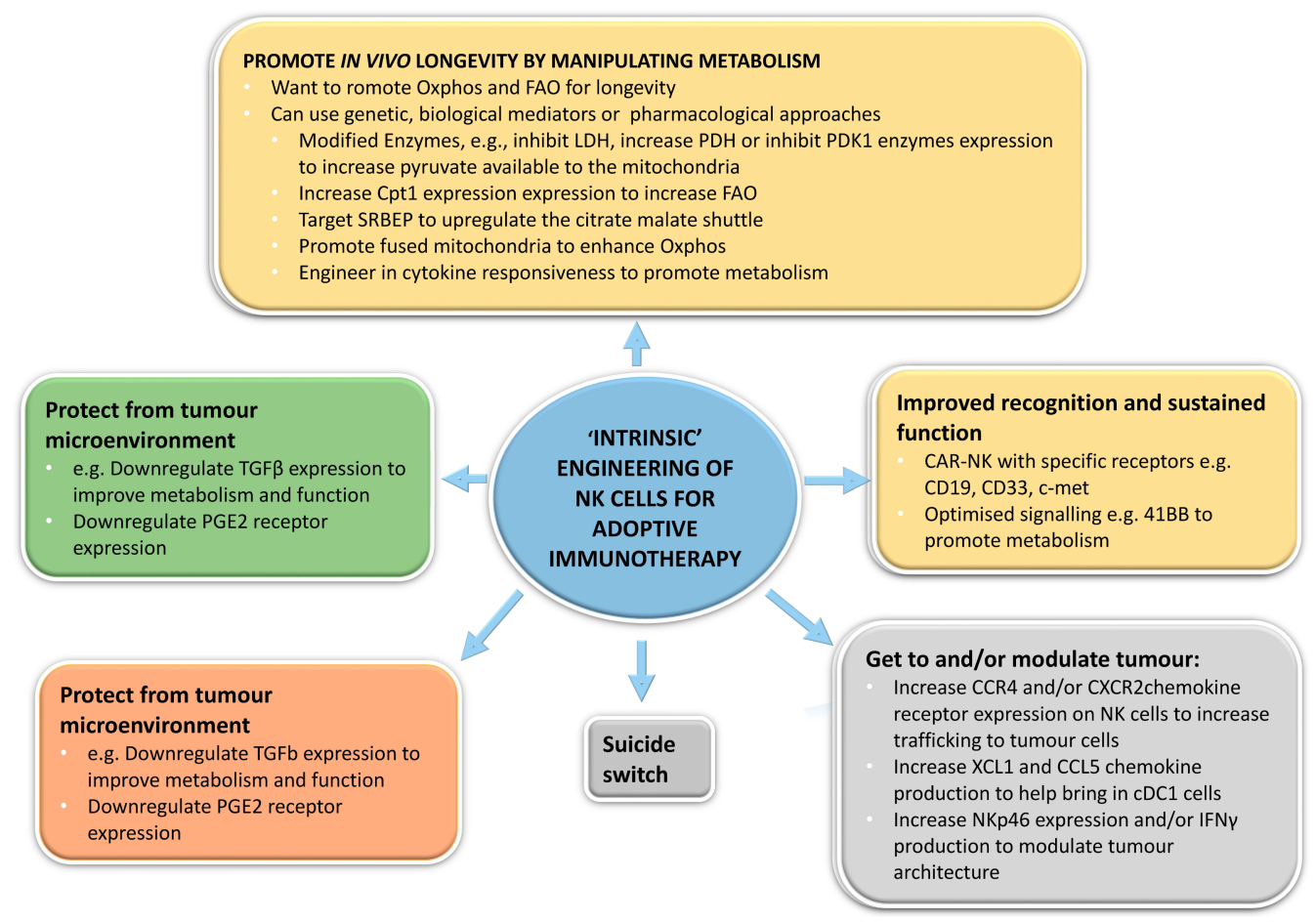

Figure 3. Intrinsic "rewiring" of NK cells for adoptive immunotherapy. NK cells, particularly allogeneic NK cells, offer huge potential for genetic engineering to improve metabolism and function of adoptively transferred NK cells for cancer immunotherapy. This figure identifies some potential mechanisms by which this can be done. 
Promote in vivo longevity: Allogeneic adoptively transferred cells will ultimately be removed by the host immune response and the kinetics of this can limit their effectiveness. Indeed, the success of NK cell adoptive cell therapy is improved by longevity and persistence of effector cells [43]. As a result, various strategies continue to be investigated including engineering IL15 into CAR-NK cells constructs, an approach which significantly increased survival of CAR-NK cells in a Raji xenograft model [44].

As metabolism is fundamentally linked with survival, it has the potential to provide many targets by which to increase NK cell longevity in vivo. Work from the $\mathrm{T}$ cell field suggests that FAO and Oxphos are generally better for promoting longevity with enhanced therapeutic potential $[45,46]$. Various strategies have been used successfully in $\mathrm{T}$ cells, including inhibition of glycolysis by 2DG but care will need to be taken when extrapolating to NK cells, given their different metabolic wiring. NK cells depend hugely on glucose which fuels both glycolysis and Oxphos in NK cells. Therefore, a global shut down of the glycolytic pathway may not be beneficial. Indeed, it has been shown that 2DG also inhibits important NK cell function in vitro and in vivo [20,47]. It may be more advantageous to target pyruvate towards mitochondrial metabolism. There are a number of enzymes that could be targeted either pharmacologically or genetically. Strategies could include inhibition of lactate dehydrogenase (LDH), enhanced activity of pyruvate dehydrogenase (PDH) or inhibition of pyruvate dehydrogenase kinase 1 (PDK1), any of which would have a net effect of shuttling pyruvate away from lactate production and towards mitochondrial metabolism.

Given that the CMS metabolic pathway appears to be prioritized by NK cells, targeting molecules that regulate this pathway is an obvious strategy. For example, increasing Srebp activity, might increase flux through this pathway, and the resulting increase in Oxphos could promote longevity of the cells. The role of FAO in NK cells has not been well described but given that FAO promotes longevity in other cells, engineering increased Cpt1 expression in NK cells could facilitate increased FAO.

One interesting concept with respect to mitochondria regards the possibility that forced alterations in their physical organization can be used to manipulate their cellular metabolism [48]. It is known that mitochondrial structural organization is a dynamic process. Cells that efficiently engage Oxphos tend to have fused mitochondria with tubular structures, while mitochondria tend to be more fragmented when cells are engaged in glycolytic metabolism. Pharmacological manipulation of therapeutic $\mathrm{T}$ cells towards a fused mitochondrial phenotype improved their longevity, function and tumour killing in a lymphoma model [48].

Other indirect mechanisms include engineering in cytokines that promote NK cell survival and metabolism and proof of principle for this approach (using IL15) has already been demonstrated in a CAR-NK preclinical model [44]. However, more work on the basic metabolism 
within NK cells and the mechanisms of regulation are required to make informed decisions. Indeed, it is likely that NK cells from different sources and environments will have different metabolic requirements that will have to be considered.

Improved recognition and sustained function: The success of CAR-T cells is beyond question but as previously mentioned, problems relating to $\mathrm{T}$ cell biology, including CRS, present significant problems. NK cells offer potential advantages over CAR-T cells, particularly with respect to treatment induced pathology. A recent study by Li et al. showed that in a direct comparison of CAR-T and CAR-NK cells in a mouse ovarian cancer xenograft model, both cell types were equally effective in terms of reducing tumour burden. However, there was significant pathology with the CAR-T cells (including CRS and weight loss) but not with CAR-NK cells [49]. Because CAR-NK cells are already being genetically engineered, there is clear potential to further improve their metabolic fitness. One approach is to optimize signaling domains included in the CAR. For example, the signaling domain of 4-1BB (CD137) has been reported to alter metabolism of cells towards increased fatty acid oxidation and Oxphos while CD28 signalling was associated with an increased glycolytic metabolism [50]. However, when various NK cell signaling domains were optimized in their CAR-NK cells, Li et al. did not find any additional benefit by including a 4-1BB domain [49].

Antagonise NK check-point inhibitors: NK cells express lots of inhibitory receptors including KIR, TIGIT, Tim 3 etc., all of which are potential targets to enhance NK cell function, particularly in a cancer environment. Downregulation of receptors or key signaling components are possible strategies to improve adoptive NK cells. This could be extended to either enhancing or suppressing cytokine receptor signaling e.g., IL15 is a strong driver of NK cell metabolism and function. Identification of CIS (encoded by Cish) as a strong negative regulator of IL15 signalling revealed it as a potential target to promote enhanced metabolism, longevity and function of NK cells in vivo [51]. Indeed, Cish(-/-) mice had enhanced IL15 signalling and resistance to metastases in prostate, melanoma and breast cancer models [51].

Protect from the tumour microenvironment: Adoptive cell therapies involve sending cells into hostile environments created by cancer. Nutrients may be in short supply. Tumours are often hypoxic and numerous mechanisms by which tumours suppress NK cells are already identified [52]. Cells could be engineered to improve survival in this environment, e.g., TGF $\beta$ is often found elevated in cancer and is a known suppressor of NK cell metabolism and function [37,38]. Similarly, PGE2 produced by tumour cells can inhibit NK cells [53]. Engineering CAR-NK or adoptive NK cells to express reduced TGF $\beta$ or PGE2 receptors might prolong their survival in vivo. In a similar fashion, downregulation of adenosine A2 receptor on CAR-NK cells, which inhibits NK cell functions, may prove beneficial as previously shown for CAR-T cells [54]. As we 
discover more about metabolite and nutrient receptor regulation of NK cell metabolism, we might be able to better prepare cells for survival, e.g., as decreased glycolysis impairs NK cell function [20,22], we could engineer NK cells to express increased glut1 expression, to make them more competitive for this important nutrient in the tumour microenvironment.

Improved tumour access and/or modulation: While the main focus of this review has been on intrinsic mechanisms of NK cell regulation, it would be foolish to disregard the importance of NK cell extrinsic mechanisms in improving NK cell therapies. Indeed, it is likely that the combined synergy will ultimately provide the greatest benefit for patients. Manipulation of the tumour microenvironment, e.g., antibody blockade of tumour derived adenosine, may improve NK cell activity [55]. Indeed, a recent report demonstrated a benefit from combined targeting of adenosine production by tumour cells with an NKG2D expressing CAR-NK cell in various tumour models [56]. Furthermore, while it is generally acknowledged that tumour infiltrating lymphocytes are a good prognostic indicator in cancer, NK cells are more likely to be found on the periphery of tumours [57,58]. Improved recruitment to tumour e.g., by MIP1a [59] could be induced by increased expression of relevant chemokine receptors e.g., CCR4 on NK cells. This principle was also recently demonstrated by CXCR2 expression which promoted NK cell migration towards renal carcinoma cells [60]. NK cells are also important in recruiting in other anti-tumour effector cells, as recently reported by Bottcher et al., where NK cell production of CCL5 and XCL1 recruited cDC1 into tumours [53] where they could mediate their anti-tumour functions. Therefore, targeting increased production of these chemokines in NK cells could enhance the overall immune response against tumours. Finally, improved longevity of NK cells could also support NK cells in their role of preventing metastatic spread of tumours, in part by modulatation of tumour architecture as recently described by Glasner et al. [61].

\section{CONCLUDING REMARKS}

The introduction of immunotherapy has changed the landscape of medical oncology. The on-going success of a range of immunotherapeutic interventions means that they are here to stay, and are likely to become increasingly used into the future. As a result, it is imperative that there is increased collaboration and communication between cancer and immunology fields. Because cancer cells have an altered metabolism, this has become a potential target for their preferential killing compared to healthy cells (reviewed in [62]). However, many of these drugs also have the potential to interfere with immune cell metabolism [63]. One example is rapamycin which, alone or in combination, is in numerous clinical trials (over 1000 registered at www.clinicaltrial.gov). Some of these include treatments that involve NK cell mediated killing of antibody coated tumours cells, e.g., anti-GD2 for neuroblastoma, anti- 
Her2 for breast cancer or anti-CD20 for lymphoma. Given that we now know that mTORC1 is critical for NK cell metabolism, and that rapamycin can inhibit NK cell metabolism and function, inclusion of rapamycin in these treatment regimens is likely to have a negative impact on immunotherapy. This potential issue was nicely illustrated in a study where JAK inhibitors (JAKi) were evaluated to target breast cancer as the JAK-STAT pathway is commonly activated in these cells. However, the drugs actually increased the metastatic burden in pre-clinical models due to inhibition of NK cell control of metastatic spread [64].

Moving to a focus on adoptive cell therapy also has the potential to circumvent the need to restore dysregulated autologous NK cell functions in patients which remains a much bigger challenge. For example, there may be scope to use engineered off-the-shelf NK cell as effectors for antibody based targeted therapies, e.g., anti-CD20 for lymphoma, or to use allo-NK cells during remission as a prophylactic strategy against metastatic disease.

In conclusion, numerous new therapies harness, manipulate and target the immune system to fight against cancer in a myriad of different ways. The success of these treatments will likely lead to their increased use, and to their becoming standard-of-care for a variety of cancers. However, we are just at the beginning of this road and the potential for continued improved therapies is likely to pave the way to cure diseases, e.g., metastatic cancers, that are currently beyond our capabilities.

\section{CONFLICTS OF INTEREST}

The author declares that they have no conflicts of interest.

\section{REFERENCES}

1. Ledford H, Else H, Warren M. Cancer immunologists scoop medicine Nobel prize. Nature. 2018;562(7725):20-1.

2. Castellarin M, Watanabe K, June $\mathrm{CH}$, Kloss CC, Posey AD Jr. Driving cars to the clinic for solid tumors. Gene Ther. 2018;25(3):165-75.

3. Shimabukuro-Vornhagen A, Godel P, Subklewe M, Stemmler HJ, Schlosser HA, Schlaak M, et al. Cytokine release syndrome. J Immunother Cancer. 2018;6(1):56.

4. Souza-Fonseca-Guimaraes F, Cursons J, Huntington ND. The Emergence of Natural Killer Cells as a Major Target in Cancer Immunotherapy. Trends Immunol. 2019;40(2):142-58.

5. Kim S, Iizuka K, Aguila HL, Weissman IL, Yokoyama WM. In vivo natural killer cell activities revealed by natural killer cell-deficient mice. Proc Natl Acad Sci U S A. 2000;97(6):2731-6.

6. Lopez-Soto A, Gonzalez S, Smyth MJ, Galluzzi L. Control of Metastasis by NK Cells. Cancer Cell. 2017;32(2):135-54.

7. Guillerey C, Huntington ND, Smyth MJ. Targeting natural killer cells in cancer immunotherapy. Nature Immunol. 2016;17(9):1025-36. 
8. Yasumura S, Lin WC, Hirabayashi H, Vujanovic NL, Herberman RB, Whiteside TL. Immunotherapy of liver metastases of human gastric carcinoma with interleukin 2-activated natural killer cells. Cancer Res. 1994;54(14):3808-16.

9. Baluna R, Vitetta ES. Vascular leak syndrome: a side effect of immunotherapy. Immunopharmacology. 1997;37(2-3):117-32.

10. Alderson KL, Sondel PM. Clinical cancer therapy by NK cells via antibodydependent cell-mediated cytotoxicity. J Biomed Biotechnol. 2011;2011:379123.

11. Cong J, Wang X, Zheng X, Wang D, Fu B, Sun R, et al. Dysfunction of Natural Killer Cells by FBP1-Induced Inhibition of Glycolysis during Lung Cancer Progression. Cell Metab. 2018;28(2):243-55·e5.

12. Bi J, Tian Z. NK Cell Exhaustion. Front Immunol. 2017;8:760.

13. Suen WC, Lee WY, Leung KT, Pan XH, Li G. Natural Killer Cell-Based Cancer Immunotherapy: A Review on 10 Years Completed Clinical Trials. Cancer Invest. 2018;36(8):431-57.

14. Cichocki F, Verneris MR, Cooley S, Bachanova V, Brunstein CG, Blazar BR, et al. The Past, Present, and Future of NK Cells in Hematopoietic Cell Transplantation and Adoptive Transfer. Curr Top Microbiol Immunol. 2016;395:225-43.

15. Mehta RS, Rezvani K. Chimeric Antigen Receptor Expressing Natural Killer Cells for the Immunotherapy of Cancer. Front Immunol. 2018;9:283.

16. Saetersmoen ML, Hammer Q, Valamehr B, Kaufman DS, Malmberg KJ. Offthe-shelf cell therapy with induced pluripotent stem cell-derived natural killer cells. Semin Immunopathol. 2019;41(1):59-68.

17. Zhang J, Zheng H, Diao Y. Natural Killer Cells and Current Applications of Chimeric Antigen Receptor-Modified NK-92 Cells in Tumor Immunotherapy. Int J Mol Sci. 2019;20(2):317.

18. Assmann N, O'Brien KL, Donnelly RP, Dyck L, Zaiatz-Bittencourt V, Loftus $\mathrm{RM}$, et al. Srebp-controlled glucose metabolism is essential for NK cell functional responses. Nature Immunol. 2017;18(11):1197-206.

19. Keppel MP, Saucier N, Mah AY, Vogel TP, Cooper MA. Activation-specific metabolic requirements for NK Cell IFN-gamma production. J Immunol. 2015;194(4):1954-62.

20. Donnelly RP, Loftus RM, Keating SE, Liou KT, Biron CA, Gardiner CM, et al. mTORC1-dependent metabolic reprogramming is a prerequisite for NK cell effector function. J Immunol. 2014;193(9):4477-84.

21. Marcais A, Cherfils-Vicini J, Viant C, Degouve S, Viel S, Fenis A, et al. The metabolic checkpoint kinase mTOR is essential for IL-15 signaling during the development and activation of NK cells. Nature Immunol. 2014;15(8):749-57.

22. Keating SE, Zaiatz-Bittencourt V, Loftus RM, Keane C, Brennan K, Finlay DK, et al. Metabolic Reprogramming Supports IFN-gamma Production by CD56 ${ }^{\text {bright }}$ NK Cells. J Immunol. 2016;196(6):2552-60.

23. Walls J, Sinclair L, Finlay D. Nutrient sensing, signal transduction and immune responses. Semin Immunol. 2016;28(5):396-407. 
24. Loftus RM, Assmann N, Kedia-Mehta N, O'Brien KL, Garcia A, Gillespie C, et al. Amino acid-dependent cMyc expression is essential for NK cell metabolic and functional responses in mice. Nat Commun. 2018;9(1):2341.

25. Filipovic I, Chiossone L, Vacca P, Hamilton RS, Ingegnere T, Doisne JM, et al. Molecular definition of group 1 innate lymphoid cells in the mouse uterus. Nat Commun. 2018;9(1):4492.

26. Freud AG, Mundy-Bosse BL, Yu J, Caligiuri MA. The Broad Spectrum of Human Natural Killer Cell Diversity. Immunity. 2017;47(5):820-33.

27. Salzberger W, Martrus G, Bachmann K, Goebels H, Hess L, Koch M, et al. Tissue-resident NK cells differ in their expression profile of the nutrient transporters Glut1, CD98 and CD71. PLoS One. 2018;13(7):e0201170.

28. Jensen H, Potempa M, Gotthardt D, Lanier LL. Cutting Edge: IL-2-Induced Expression of the Amino Acid Transporters SLC1A5 and CD98 Is a Prerequisite for NKG2D-Mediated Activation of Human NK Cells. J Immunol. 2017;199(6):1967-72.

29. Schafer JR, Salzillo TC, Chakravarti N, Kararoudi MN, Trikha P, Foltz JA, et al. Education-dependent activation of glycolysis promotes the cytolytic potency of licensed human natural killer cells. J Allergy Clin Immunol. 2019;143(1):346-58

30. Pfeifer C, Highton AJ, Peine S, Sauter J, Schmidt AH, Bunders MJ, et al. Natural Killer Cell Education Is Associated With a Distinct Glycolytic Profile. Front Immunol. 2018;9:3020.

31. Cichocki F, Wu CY, Zhang B, Felices M, Tesi B, Tuininga K, et al. ARID5B regulates metabolic programming in human adaptive NK cells. J Exp Med. 2018;215(9):2379-95.

32. O'Sullivan D, van der Windt GJ, Huang SC, Curtis JD, Chang CH, Buck MD, et al. Memory CD8(+) T cells use cell-intrinsic lipolysis to support the metabolic programming necessary for development. Immunity. 2014;41(1):75-88.

33. Anderson KA, Hirschey MD. Mitochondrial protein acetylation regulates metabolism. Essays Biochem. 2012;52:23-35.

34. Ren J, Panther E, Liao X, Grammer AC, Lipsky PE, Reilly CM. The Impact of Protein Acetylation/Deacetylation on Systemic Lupus Erythematosus. Int J Mol Sci. 2018;19(12):4007.

35. O'Brien KL, Finlay DK. Immunometabolism and natural killer cell responses. Nat Rev Immunol. 2019;19(5):282-90. doi: 10.1038/s41577-019-0139-2

36. Felices M, Lenvik AJ, McElmurry R, Chu S, Hinderlie P, Bendzick L, et al. Continuous treatment with IL-15 exhausts human NK cells via a metabolic defect. JCI Insight. 2018;3(3):e96219.

37. Viel S, Marcais A, Guimaraes FS, Loftus R, Rabilloud J, Grau M, et al. TGFbeta inhibits the activation and functions of NK cells by repressing the mTOR pathway. Sci Signal. 2016;9(415):ra19.

38. Zaiatz-Bittencourt V, Finlay DK, Gardiner CM. Canonical TGF-beta Signaling Pathway Represses Human NK Cell Metabolism. J Immunol. 2018;200(12):3934-41. 
39. Yang M, Chen S, Du J, He J, Wang Y, Li Z, et al. NK cell development requires Tsc1-dependent negative regulation of IL-15-triggered mTORC1 activation. Nat Commun. 2016;7:12730.

40. Yang C, Tsaih SW, Lemke A, Flister MJ, Thakar MS, Malarkannan S. mTORC1 and mTORC2 differentially promote natural killer cell development. Elife. 2018;7:e35619. doi: 10.7554/eLife.35619

41. Wang F, Meng M, Mo B, Yang Y, Ji Y, Huang P, et al. Crosstalks between mTORC1 and mTORC2 variagate cytokine signaling to control NK maturation and effector function. Nat Commun. 2018;9(1):4874.

42. Castro W, Chelbi ST, Niogret C, Ramon-Barros C, Welten SPM, Osterheld K, et al. The transcription factor Rfx7 limits metabolism of NK cells and promotes their maintenance and immunity. Nat Immunol. 2018;19(8):809-20.

43. Bachanova V, Cooley S, Defor TE, Verneris MR, Zhang B, McKenna DH, et al. Clearance of acute myeloid leukemia by haploidentical natural killer cells is improved using IL-2 diphtheria toxin fusion protein. Blood. 2014;123(25):3855-63.

44. Liu E, Tong Y, Dotti G, Shaim H, Savoldo B, Mukherjee M, et al. Cord blood NK cells engineered to express IL-15 and a CD19-targeted CAR show long-term persistence and potent antitumor activity. Leukemia. 2018;32(2):520-31.

45. Sukumar M, Kishton RJ, Restifo NP. Metabolic reprograming of anti-tumor immunity. Curr Opin Immunol. 2017;46:14-22.

46. Crompton JG, Sukumar M, Roychoudhuri R, Clever D, Gros A, Eil RL, et al. Akt inhibition enhances expansion of potent tumor-specific lymphocytes with memory cell characteristics. Cancer Res. 2015;75(2):296-305.

47. Mah AY, Rashidi A, Keppel MP, Saucier N, Moore EK, Alinger JB, et al. Glycolytic requirement for NK cell cytotoxicity and cytomegalovirus control. JCI Insight. 2017;2(23):95128. doi: 10.1172/jci.insight.95128

48. Buck MD, O'Sullivan D, Klein Geltink RI, Curtis JD, Chang CH, Sanin DE, et al. Mitochondrial Dynamics Controls $\mathrm{T}$ Cell Fate through Metabolic Programming. Cell. 2016;166(1):63-76.

49. Li Y, Hermanson DL, Moriarity BS, Kaufman DS. Human iPSC-Derived Natural Killer Cells Engineered with Chimeric Antigen Receptors Enhance Anti-tumor Activity. Cell Stem Cell. 2018;23(2):181-92·e5.

50. Kawalekar OU, O'Connor RS, Fraietta JA, Guo L, McGettigan SE, Posey AD, Jr., et al. Distinct Signaling of Coreceptors Regulates Specific Metabolism Pathways and Impacts Memory Development in CAR T Cells. Immunity. 2016;44(2):380-90.

51. Delconte RB, Kolesnik TB, Dagley LF, Rautela J, Shi W, Putz EM, et al. CIS is a potent checkpoint in NK cell-mediated tumor immunity. Nat Immunol. 2016;17(7):816-24.

52. Chambers AM, Lupo KB, Matosevic S. Tumor Microenvironment-Induced Immunometabolic Reprogramming of Natural Killer Cells. Front Immunol. 2018;9:2517.

53. Bottcher JP, Bonavita E, Chakravarty P, Blees H, Cabeza-Cabrerizo M, Sammicheli S, et al. NK Cells Stimulate Recruitment of cDC1 into the Tumor 
Microenvironment Promoting Cancer Immune Control. Cell. 2018;172(5):1022-37·e14.

54. Beavis PA, Henderson MA, Giuffrida L, Mills JK, Sek K, Cross RS, et al. Targeting the adenosine $2 \mathrm{~A}$ receptor enhances chimeric antigen receptor $\mathrm{T}$ cell efficacy. J Clin Invest. 2017;127(3):929-41.

55. Young A, Ngiow SF, Barkauskas DS, Sult E, Hay C, Blake SJ, et al. Coinhibition of CD73 and A2AR Adenosine Signaling Improves Anti-tumor Immune Responses. Cancer Cell. 2016;30(3):391-403.

56. Wang J, Lupo KB, Chambers AM, Matosevic S. Purinergic targeting enhances immunotherapy of CD73(+) solid tumors with piggyBac-engineered chimeric antigen receptor natural killer cells. J Immunother Cancer. 2018;6(1):136.

57. Mamessier E, Sylvain A, Thibult ML, Houvenaeghel G, Jacquemier J, Castellano R, et al. Human breast cancer cells enhance self tolerance by promoting evasion from NK cell antitumor immunity. J Clin Invest. 2011;121(9):3609-22.

58. Carrega P, Morandi B, Costa R, Frumento G, Forte G, Altavilla G, et al. Natural killer cells infiltrating human nonsmall-cell lung cancer are enriched in CD56 bright CD16(-) cells and display an impaired capability to kill tumor cells. Cancer. 2008;112(4):863-75.

59. Glas R, Franksson L, Une C, Eloranta ML, Ohlen C, Orn A, et al. Recruitment and activation of natural killer (NK) cells in vivo determined by the target cell phenotype. An adaptive component of NK cell-mediated responses. J Exp Med. 2000;191(1):129-38.

60. Kremer V, Ligtenberg MA, Zendehdel R, Seitz C, Duivenvoorden A, Wennerberg E, et al. Genetic engineering of human NK cells to express CXCR2 improves migration to renal cell carcinoma. J Immunother Cancer. 2017;5(1):73.

61. Glasner A, Levi A, Enk J, Isaacson B, Viukov S, Orlanski S, et al. NKp46 Receptor-Mediated Interferon-gamma Production by Natural Killer Cells Increases Fibronectin 1 to Alter Tumor Architecture and Control Metastasis. Immunity. 2018;48(2):396-8.

62. Luengo A, Gui DY, Vander Heiden MG. Targeting Metabolism for Cancer Therapy. Cell Chem Biol. 2017;24(9):1161-80.

63. Buck MD, Sowell RT, Kaech SM, Pearce EL. Metabolic Instruction of Immunity. Cell. 2017;169(4):570-86.

64. Bottos A, Gotthardt D, Gill JW, Gattelli A, Frei A, Tzankov A, et al. Decreased NK-cell tumour immunosurveillance consequent to JAK inhibition enhances metastasis in breast cancer models. Nat Commun. 2016;7:12258.

How to cite this article:

Gardiner CM. NK Cell Metabolism and the Potential Offered for Cancer Immunotherapy. Immunometabolism. 2019;1:e190005. https://doi.org/10.20900/immunometab20190005 